\title{
3 Possibilism
}

In the last chapter, we looked at the simple conditional analysis, which has its roots in the literature on voluntary actions and free will. In this chapter, we will look in detail at the other one of those two major views: the view that an agent has an ability to $\phi$ if and only if it is possible, in a properly restricted sense, for the agent to $\phi$. I will refer to that view as "possibilism" in what follows.

Possibilism has its roots in the quest for a formal understanding of the modal auxiliaries, such as "must", "might", and - most crucially for our purposes - "can". The view emerged primarily in the course of the attempt to give a unified semantic and logical account of modal auxiliaries and has played a major role in the more formal regions of philosophy, as well as in logics and linguistics. Thanks to influential proponents, such as the linguist Kratzer $(1977,1981)$ and the logician von Wright (1951), possibilism is now the point of reference for most people with a background in those areas. ${ }^{1}$ Interestingly, counterfactuals, or indeed conditionals in general, do not seem to play any role in those more formally oriented attempts to analyze ability statements. Instead, "can" statements in general, and a forteriori "can" statements expressing ability, are viewed as categorical statements about what is restrictedly possible for the agent to do.

To get a grip on possibilism, the natural place to start is Kratzer's modal semantics. In the next section, 3.1, I will give an outline of the crucial elements of that semantics, with a focus on "can" statements. In section 3.2, I will show how ability statements enter the picture; here, possibilism is introduced more formally. With the firm understanding of the view at hand, we can then move on to the discussion of possibilism. As we will see in sections 3.3 to 3.6, possibilism looks somewhat less problem-fraut than the simple conditional analysis in some ways, but, and this is the major import from the chapter, it ultimately fails as well - the truth conditions of ability statements are not properly stated in terms of restricted possibility. By the end of the chapter, we will have learned enough about abilities and the challenges in capturing them to be prepared to tackle the task of coming up with a superior view.

1 Even though possibilism has its roots in the formal treatment of modal auxiliaries, it has not been limited to those areas. In the philosophical literature, proponents of the view have applied possibilism to the problem of time travel (Lewis 1976) and also, but less famously, to the problem of free will (Lehrer 1976; Horgan 1979), where possibilism has never quite managed to make it to center stage.

○ OpenAccess. (c) 2020 Jaster, published by De Gruyter. (cc) BY-NC-ND This work is licensed under the Creative Commons Attribution-NonCommercial-NoDerivatives 4.0 License.

https://doi.org/10.1515/9783110650464-004 


\subsection{Modal semantics: Restricted possibility}

In most areas of linguistics, “can” statements are taken to exhibit a unified semantics. According to that semantics, which has been developed in most detail by Kratzer (1977, 1981), “can” is a sentence operator which takes a proposition as its argument and forms a new proposition from it. "Pigs fly", for instance, can be taken as an argument for claims such as "Pigs can fly" or, in the negated form, "Pigs cannot fly". The structure of such statements is CAN(Pigs fly) and $\neg$ CAN(Pigs fly), respectively.

What does the CAN operator do? It assigns possibility to the proposition over which it has scope. "Pigs can fly" states that it is possible that pigs fly. "Pigs cannot fly" states that it is not possible that they fly. CAN(Pigs fly) is thus equivalent to some statement of the form $\diamond$ (Pigs fly), where the diamond is the possibility operator.

What is it for something to be possible? To say that something is possible, according to the standard semantics, is for there to be a possible world in which that thing is the case. In other words, it is to apply existential quantification to the possible worlds. For it to be possible that pigs fly, say, there has to be a possible world in which pigs fly. To make a statement of the form CAN(p), then, is to state a possibility, the possibility that $\mathrm{p}$. In possible worlds terminology, the truth conditions for such a statement are that there is a possible world in which $\mathrm{p}$.

The kind of quantification that is applied to possible worlds is called the modal force of a modal expression. Expressions like "can" or "might" express possibility: the modal force of such expressions is existential quantification. For a possibility statement to be true, there has to be at least one world among the possible worlds in which the proposition over which the modal ranges is true.

Expressions like "must" or "ought", in contrast, express necessity. The modal force of such expressions is universal quantification. For a necessity statement to be true, all possible worlds have to be such that the sentence over which the modal ranges is true in them. Since we are concerned with Kratzer's semantics in connection with the idea that ability ascriptions express possibilities, the modal force we are going to be concerned with throughout the chapter will be existential quantification.

So much for the very basic framework. Let us now move on to the more complex bits. The complexity comes about in virtue of the fact that possibility statements (and modal statements in general) are virtually never unqualified - when we say that something is possible and thereby state that there is a possible world in which that thing is the case, we usually do not usually quantify over the totality of all worlds, but rather over a certain subset of those worlds - Kratzer calls 
this subset the modal base of the modal. In other words, possibility statements are usually statements of what we can call restricted possibility. When we think about whether $\mathrm{p}$ is possible, we are thinking about whether $\mathrm{p}$ is the case in some of the worlds that matter in our context. Let's call the worlds in the modal base the relevant worlds. (I'll use the terms "modal base" and "relevant worlds" interchangeably throughout the book.)

Which worlds are relevant is a matter of the conversational background, according to Kratzer. Where Kratzer speaks of the conversational background, I'll speak of a context. The idea is the same. It is simply that

when a botanist says, in a foreign country with unfamiliar vegetation, 'Hydrangeas can grow here', she says not just that in some metaphysically possible worlds there are hydrangeas growing on this soil. She speaks only about worlds where the biology of hydrangeas, the geology and the climate of the country are as they are in actuality. When a detective says, 'Mary [cannot] be the murderer', she speaks about a different set of worlds: those that are compatible with everything she knows. In each case the conversational background selects a set of propositions; propositions about the actual facts of biology and geology, or the propositions that are known to the detective. The modal base is the set of worlds in which those propositions are true. (Vetter 2015: 68)

On Kratzer's view, then, “can” and other modals are actually used relationally: there is no absolute "can", but rather always a relational "can in view of". This relative modal phrase has two arguments: the first argument is provided by a phrase which specifies the facts in view of which it is true to say of someone that she can perform a given act - the set of propositions which determine the modal base. It is provided by the meaning of a phrase like "the biology of hydrangeas and the features of the soil", or "what the detective knows". The second argument is provided by the meaning of a sentence like "Hydrangea grow here", or "Fred is the murderer" (cf. Kratzer 1977: 341). It specifies what can be the case in view of the facts specified in the first argument.

The "in view of" locution will prove very handy in the course of the book. It is a good means to express, in ordinary language, that we are talking about a restricted realm of possible worlds. I will often use phrases of the form " $\mathrm{S}$ can $\phi$ in view of p", and when I do so, I use the "in view of" phrase in the very way Kratzer intends it: as a means to express that we are restricting the possible worlds in accordance to the facts specified by "p". The "in view of" locution provides the means to pick out the modal base worlds in a non-technical fashion.

Before we look more closely at some of the details of Kratzer's semantics, let's summarize the basic idea briefly. Kratzer offers an account of "can" statements, such as "Hydrangeas can grow here". Her fundamental insight is that any such statement carries a (typically implicit) "in view of p" supplement. To 
say that hydrangeas can grow here, in some context, is to say that they can, in view of some set of facts, grow on whatever place is referred to by "here" in that context. The "in view of" locution is to be interpreted in terms of compossibility. To say that hydrangeas can grow here is to say that the growth of hydrangeas on this spot is compossible with the features of the soil, the biology of hydrangeas, and whatever else matters to us in the context. To say that the two facts are compossible is to say that there is a relevant world - a world in which the soil and the biology of hydrangeas is as in actuality - in which hydrangeas grow.

A note on terminology. In what follows, I will often say that we hold certain facts fixed or constant across possible worlds or modal space. I find this a handy way of saying that certain facts go into the modal base, or, to put it differently still, that the relevant worlds are worlds in which those very facts obtain.

I will also often say of certain facts that they get varied across the possible worlds or across modal space. I use this locution as a means to put emphasis on the fact that certain features of the actual world are irrelevant for the determination of the relevant worlds, which form the modal base. When I say that hydrangeas can grow here, for instance, lots of features of the actual world are varied: my body weight, who the president of the United States is, and usually also whether or not there is a car standing right on the spot where it is said to be possible for the hydrangeas to grow. It is important to note that this talk about fixation and variation of facts does not add anything to Kratzer's framework, nor does it alter anything about it. It is merely a different way of putting things.

So much for the overview. Let us now proceed to some more subtle parts of the semantics. In particular, there are two aspect I would like to highlight, because they will prove helpful in embedding the possibilist semantics of ability statements in the possibilist semantics of "can" statements quite generally. The first is that there are generally two kinds of modal bases. Vetter's examples above illustrate this nicely. On the one hand, there are so-called circumstantial bases. Here, the relevant worlds are determined by propositions about facts in the world which do not depend on our epistemic access to them. The statement "Hydrangeas can grow here" is an example of a statement with a circumstantial base. The modal base contains facts about biology, the soil, and so on. "Pigs can't fly" can also be read as a statement about circumstantial possibility. It is naturally read as the statement that in view of their anatomy, their lack of wings and so forth, pigs cannot fly.

On the other hand, there are so-called epistemic bases. Here, the relevant worlds are fixed by facts we have knowledge about. "John cannot be the murderer” is naturally read along those lines. It is naturally read as the statement that, in view of what the detective knows about the crime and John's whereabouts, it is not possible that John be the murderer. Note that "Pigs can't fly" can also be 
read as a statement about epistemic possibility: in view of what we know about pigs, they cannot fly.

Both circumstantial possibility and epistemic possibility are realistic. That is to say that the actual world is always contained in the modal base. The reason for that is that the facts which determine which worlds count as relevant in a given context - the features of the world we hold fixed across possible worlds are always that: features of the actual world. And since any world in which those facts obtain go into the modal base, the actual world will always automatically be among them. Epistemic possibility does not differ from circumstantial possibility in this respect. Since knowledge is factive - we can only know $p$, if $p$ is true - the set of worlds in which the things we know are held fixed will always automatically contain the actual world.

Let's now move on to the second more subtle feature of Kratzer's framework. So far, I have introduced one important role of the context, or the conversational background, as Kratzer calls it: the context fixes a set of relevant worlds for possibility statements. Differently put, it determines a set of facts which are then held fixed across possible worlds. Something is possible, in the correspondingly restricted sense, if the so determined relevant worlds contain a world in which that thing is the case.

But this is not the only role the context plays in Kratzer's framework. The context also determines the so-called ordering source. As Kratzer observes, modal expressions are gradable. Some things are more easily possible than others. For some things there is a good possibility, for others merely a slight possibility. And so on.

The modal base is the wrong tool to account for this kind of graded modals. To account for the fact that there is a better possibility for Kim to be the murderer than there is a possibility for Jim to be the murderer, it will not do to check whether or not the modal base contains a world in which Kim is the murderer and whether or not it contains one in which Jim is the murderer. If each agent can be the murderer in view of what we know, say, then we will find both a world in which Kim is the murderer and one in which Jim is the murderer among the worlds in which we hold fixed what we know. To account for the fact that there is a better possibility for the one than for the other, we need a means to establish a scale of some kind.

The ordering source is designed to do just that (Kratzer 1981: 46f.). It ranks the relevant worlds in accordance to their closeness to some ideal. Ordering sources come in different kinds. A bouletic ordering source ranks worlds by how close they are to fully satisfying the speaker's desires. A deontic sources ranks worlds by how close they come to meeting the demands of morality or the 
law. A stereotypical ordering source ranks worlds by how close they are to being entirely normal.

We can think of the ordering source as a set of propositions, which together express some ideal. Roughly, a world is closer to the ideal the more propositions of the ideal are true in it. A world in which all the laws are respected, for instance, is closer to the deontic ideal than a world in which some or all laws are broken.

That there is a better possibility for Kim being the murderer than there is one for Jim being the murderer will presumably be a matter of a stereotypical ordering source. The ideal is a world in which everything is normal. Worlds in which Jim is the murderer are less extraordinary than worlds in which Kim is the murderer. Thus, there is a better possibility for the former than for the latter, despite the fact that there is some possibility for each.

\subsection{Possibilism: The details}

Against the background of this overview, we can now return to our original topic: the understanding of ability statements in terms of restricted possibilities as it is suggested by possibilists. Probably the best-known expression of possibilism in the philosophical literature can be found in the following famous passage by Lewis:

To say that something can happen means that its happening is compossible with certain facts. Which facts? That is determined (...) by context. An ape can't speak a human language - say, Finnish - but I can. Facts about the anatomy and operation of the ape's larynx and nervous system are not compossible with his speaking Finnish. The corresponding facts about my larynx and nervous system are compossible with my speaking Finnish. But don't take me along to Helsinki as your interpreter: I can’t speak Finnish. My speaking Finnish is compossible with the facts considered so far, but not with further facts about my lack of training. What I can do, relative to one set of facts, I cannot do, relative to another, more inclusive, set. (Lewis 1976: 149)

In this passage, Lewis formulates the sketch of a view of abilities which treats ability statements as restricted possibility statements. What we do, when we ascribe the ability to $\phi$ to an agent, according to Lewis, is quantify existentially over a restricted domain of relevant possible worlds and postulate that the agent $\phi$ 's in at least one of those worlds.

To say that ability statements express possibility is to say, in Kratzer's terminology, that the modal force of ability statements is that of existential quantification. This is the core claim of possibilism. The interesting question is: what is 
their modal base? What is obvious is that the modal base will have to be circumstantial, rather than epistemic. What matters for an agent's abilities is what the agent can do in view of certain facts in the world; the question is not what she can do in view of what we know about those or any other facts.

But which facts matter? Here, Lewis suggests a contextualist view: which facts go into the modal base - which features of the world are held fixed across modal space - depends on the speaker context. Sometimes, we may be interested in what Lewis can do in view of his anatomy. Sometimes, we may be interested in what he can do in view of his amount of training. Depending on the context, it will vary which facts go into the modal base.

Kratzer, whose work contains only a few scattered remarks specifically about ability ascriptions, suggests the very same picture. In the most explicit passage I know of, she considers the sentence "Ich kann nicht Posaune spielen" ("I cannot play the trombone") and writes:

Depending on the situation in which I utter this sentence, I may say quite different things. I may mean that I don't know how to play the trombone. I am sure that there is something in a person's mind which becomes different when he or she starts learning how to play the trombone. A programme is filled in. And it is in view of this programme that it may be possible that I play the trombone. Or suppose that I suffer from asthma. I can hardly breathe. In view of my physical condition I am not able to play the trombone, although I know how to do it. I may express this by uttering ["Ich kann nicht Posaune spielen”]. Or else imagine that I am travelling by sea. The ship sinks and so does my trombone. I manage to get to a lonely island and sadly mumble ["Ich kann nicht Posaune spielen"]. I could play the trombone in view of my head and my lungs, but the trombone is out of reach. (Kratzer 1981: 54)

Kratzer and Lewis both endorse a contextualist account of ability statements, then. The modal base of ability statements varies in accordance to the context in which the statement is uttered. Again, the same picture is laid out by Horgan, an almost entirely forgotten proponent of possibilism. As Horgan writes in a brilliant paper on free will,

[w] hen we say that (...) someone could have done something he did not actually do, we are saying that it was possible for the given (...) action to occur, even though it did not actually occur. I.e., we are asserting a statement of the form $\diamond \phi$, where $\diamond$ is the possibility-operator of modality. And according to contemporary semantical treatments of modality, $\diamond \phi$ is true if and only if $\phi$ is true in some possible world that is accessible from the actual world. So if we wish to apply possible-world semantics to everyday "could"-statements, the key question we face is this: What is the relevant notion of accessibility? I suggest that the accessible possible worlds are those in which the circumstances are sufficiently similar to the circumstances which prevailed in the actual world. (...) The notion of circumstantial similarity is obviously quite vague (...). We resolve the vagueness (...) in varying ways, depending on the 
context in which a given "could"-statement is used and the speaker's purposes in using it. (Horgan 1979: 346)

Lewis, Kratzer, and Horgan all voice the same idea: ability ascriptions have different modal bases, depending on the context of utterance. ${ }^{2}$

We are now in a position to formulate the possibilist's view a bit more precisely. In the beginning, we said that an agent has an ability, according to possibilism, if and only if it is possible, in a properly restricted sense, for the agent to $\phi$. More specifically, though, possibilism is an explicitly contextualist view about ability statements: it is the view that an agent $S$ has an ability to $\phi$ if and only if it is possible, in the properly restricted sense, for the agent to $\phi$, where the proper restrictions will vary across contexts. ${ }^{3}$ Or, to put it in possible worlds terminology,

POSS. an agent $\mathrm{S}$ has an ability to $\phi$ if and only if there is a relevant world in which the agent $\phi$ 's, where the set of the relevant worlds will vary across ascriber contexts.

It is the contextualist aspect of possibilism in particular, I take it, that makes the view extremely appealing. Ability statements do in fact seem to exhibit a certain context sensitivity that seems to have to do with our focus on certain features of the agent and facts of her situation. Can I jump the fence? Well, in a sense I can: I have done it many times in the past and it was easy. In a different sense, I cannot: my leg is currently broken. Can Emma kill her husband? In a sense, she certainly can: she owns a gun and knows very well how to use it. In a different sense, she cannot: she has tried it before when her husband was asleep and never managed to actually pull the trigger. Can Fred play the piano? In a sense, yes: he has had lessons since he was five years old. In another sense,

2 One should not be distracted by the fact that Horgan operates with the notion of circumstantial similarity. As Lewis (1981) shows in connection with counterfactuals, there is no substantial difference between what he calls "ordering semantics" - a semantics which works with restrictions to the similar worlds - and "premise semantics" - a semantics which works with restrictions to worlds in which the relevant facts obtain.

3 The explicitly contextualist formulation of the view adds something important to the original formulation: the original formulation leaves it open whether or not it varies which facts are held constant. Compare nomological possibility, for instance, to see this: $\mathrm{p}$ is nomologically possible if and only if there is a relevant world in which $\mathrm{p}$ is the case. But the relevant worlds will always be the worlds in which the laws of nature are held constant. In the case of abilities, there is no such general criterion to determine the relevant worlds. The overtly contextualist formulation of the view makes this fact explicit. 
he cannot: he once had a blackout on stage and ever since his fingers grow stiff when he sits down to play.

These examples show very clearly that possibilism voices a very important insight. There is definitely something to the idea that abilities are always had in view of certain facts, to use Kratzer's terminology once more. It is in view of my stable intrinsic features that I can jump the fence, and in view of my broken leg that I cannot. It is in view of Emma's owning and mastering her gun that she can kill her husband, and in view of her personality that she cannot. It is in view of Fred's amount of training that he can play the piano, and in view of his trauma that he cannot.

Possibilism spells this out in terms of the restrictions we impose on the possible worlds. Sometimes, we hold only the agent's stable intrinsic features fixed and vary her current state; sometimes, however, her current state is held fixed as well. Sometimes, we hold only the agent's possession and her mastery of it fixed and vary her personality traits; sometimes, however, we hold the personality traits fixed, too. Sometimes, we hold only the agent's amount of training fixed and vary psychological impediments; sometimes, however, we hold the psychological impediments fixed as well. Depending on the modal base, the truth conditions of ability statements vary in the right way to account for our ordinary practice of ability ascription.

Possibilism is therefore not just attractive as a formal framework, which allows us to embed ability statements into a more general formal system encompassing “can" statements quite generally. Rather, it is also a way of understanding and systematizing the variances in the truth conditions of ability statements in ordinary language.

What about the ordering source? According to Kratzer, the ordering source is empty in the case of disposition statements, and the same, I take it, goes for ability statements (Kratzer 1981: 64). Whether this is really true need not concern us at the moment. We'll get back to this when we talk about degrees of abilities ( $\rightarrow$ 3.5). For now, let's just leave it at that.

We are now equipped with a rather solid understanding of possibilism and the formal framework underneath it. Thus armored, let us now dive into the discussion of the view. Here is a road map of the following sections.

The next two sections discuss a few up- and down-sides of possibilism. In the next section, 3.3, we'll see that the restrictions to varying modal bases will prove very fruitful in the handling of some of the issues that proved problematic for the simple conditional analysis. Thanks to the possibilist's fundamental insight that abilities are always had in view of certain facts and that the facts that matter vary across contexts, possibilism has no trouble at all with masks, for instance, and it provides a major leap towards an understanding of the dif- 
ference between general and specific abilities. Here, the view has some obvious merits. In the course of the section, however, we will also see that possibilism clearly fails as an account of general abilities. The possibilist condition is simply insufficient for the having of such an ability. Just as the simple conditional analysis, possibilism exhibits a severe shortcoming in this regard.

Section 3.4 takes an equally balanced perspective. On the one hand, we are going to see that there are some obvious merits emerging from the fact that possibilism is somewhat simpler than the simple conditional analysis. Since possibilism does not focus on a modal tie between intentions and performances, but only on the possibility of the performance itself, possibilism does not have problems with impeded intentions and non-agentive abilities. Yet, we are also going to see that that simplicity comes at a price. The possibilist encounters serious trouble in delineating the boundary between abilities and other statements of restricted possibility. Possibilism may actually be a bit too simplistic when it comes to setting abilities apart.

Sections 3.5 and 3.6 focus on further problems with the view. In section 3.5, I will argue that possibilism fails to account for degrees of abilities and the context sensitivity of ability ascriptions that attaches to their gradable nature. In section 3.6, finally, I will side with Kenny (1976) in arguing that possibilism fails on formal grounds as well. Taken together, the problems outlined in the following sections are going to show very vividly that possibilism, while highly promising at the outset, turns out to be just as problematic as the simple conditional analysis on closer inspection.

\subsection{Upsides \& downsides I - General vs. specific abilities \& masks}

One reason why possibilism looks more promising than the simple conditional analysis at first is that the view seems to bring us a lot closer to understanding the difference between general and specific abilities and offers a neat understanding of the workings of masks. These are the upsides I will talk about in this section. The downside, however, will follow suit. As we will see in the last bit of the section, possibilism fails as an account of general abilities. The condition it states is far too weak here.

Let's start by focusing on the promising perspective on the distinction between general and specific abilities that is delivered by possibilism. According to the possibilist, an agent has an ability to $\phi$ if and only if there is a relevant world in which the agent $\phi$ 's. The important notion with respect to the distinction between general and specific abilities is that of relevance: which worlds 
count as relevant in a given context varies. These variances, the possibilist can argue, generate abilities that are sometimes general, and sometimes specific.

Without using the terminology of general and specific abilities, Kratzer is quite explicit on the fact that she thinks of the distinction between general and specific abilities along those lines. Recall that, on her view, a speaker, call her Angelika, may say different things by uttering sentences like "Ich kann nicht Posaune spielen" ("I cannot play the trombone"). On the one hand, Angelika may express that she does not know how to play the trombone. On the other hand, she may express that the fact that she can hardly breathe or the fact that no trombone is available prevents her from doing it. As is easy to see, what Kratzer points at in distinguishing these uses of the sentence is the same distinction we recognized by the terms "general abilities" and "specific abilities". If one does not know how to play, one lacks a general ability to play. If one does not have a trombone available or has trouble breathing, one lacks a specific ability.

The way the distinction is accounted for is in terms of varying modal bases, or, put another way, of varying sets of relevant worlds. When we think about the general ability to play the trombone, we hold only certain intrinsic facts about Angelika's brain structure fixed - the "programme [that] is filled in" (Kratzer 1981: 54) when she learns how to play the trombone. When we think about the specific ability to play the trombone, in contrast, we also hold facts about her particular situation fixed: her breathing problems, for instance, or her current lack of a trombone.

This implies that, taken out of context, the question "Can Angelika play the trombone?” does not admit of a simple yes-or-no answer. In one sense, she can. In a different sense, she cannot. She has the general ability, but lacks the specific one. The possibilist does not seem to have trouble with any of this. Both Angelika's general ability to play the trombone and her lack of the specific ability to do it seem to be accounted for by the possibilist's framework very smoothly. They are both abilities in the sense that they can be accommodated by the same analysis. Quite generally, having an ability, according to the possibilist, is for it to be restrictedly possible for the agent to $\phi$. General and specific abilities just differ with respect to the kinds of facts that go into their respective modal bases. Possibilism thus promises a unified account of both kinds of abilities.

By the same token, possibilism offers a neat understanding of masking. A masked ability, recall, is an ability which is present, but whose exercise would be interfered with. The interfering factor, we said, is a mask. My ability to swim may be masked by a cramp. My ability to sing a certain song may be masked by my hoarseness or my shyness or my forgetfulness of the lyrics. My ability to hit the bull's eye may be masked by my sudden dizziness. 
The problem for the simple conditional analysis, recall, was that in masking cases, the counterfactual is false. It is not true that I would swim or sing or hit the bull's eye, if I intended to do so, because the closest intention worlds will be worlds in which the mask will be present. In the closest worlds in which I form the intention, I thus fail. Yet, I do have the relevant abilities. Hence, masking cases show that the truth of the counterfactual is not necessary.

Possibilism does not run into the same problem. When we are evaluating whether or not someone has the ability to swim, say, we are not moving into the closest intention worlds and seeing what happens there. Instead, we are restricting the possible worlds to the ones in which the relevant facts obtain and see whether or not there is one among them in which the agent swims. The important point with respect to masks is that, as long as the mask is not among the relevant facts, the modal base will comprise lots of worlds in which the mask is not present. Applied to our example: as long as we are not interested in the ability to swim in view of the current cramp, but simply in the general ability to swim - the ability to swim in view of one's fitness, say -, there will be quite a few worlds among the modal base in which the agent does not have a cramp and will thus swim. Possibilism is therefore quite apt to account for the fact that abilities can be had, and yet interfered with. They can be had in view of one set of facts, while their exercise would be interfered with by a different set of facts.

In chapter 2.2, when we talked about the problems the simple conditional analysis faces with respect to the distinction between general and specific abilities, we saw that the topic of general and specific abilities and the topic of masked abilities are connected. It is very obvious that general abilities can be masked, but unclear whether the same holds for specific abilities. Against the background of the preceding paragraphs, we can note that possibilism can make perfect sense of the controversy about the maskability of specific abilities. It promises a somewhat scalar account of more or less specific and general abilities. Let me explain.

Take the specific ability to do a handstand here and now. Does an agent who is, here and now, extremely scared of doing a handstand nevertheless have the specific ability to do a handstand here and now? Some may want to say she does - despite her fear, she has what it takes; her fear is just a mask. Others may want to say she doesn't. Given that she is as fearful as she is, she genuinely cannot do it. Her fear is not a mask, on that view, but actually deprives her of the specific ability.

For a similar example, let us ask: does the agent have or lack the specific ability to do a handstand here and now if, although nothing inhibits the agent's exercise of her general ability to do a handstand on the macro level, the micro 
facts happen to be such that something will interfere with the agent's exercise of doing a handstand? Suppose, for instance, a hyperacidity prevails in the agent's muscles so that any attempt to do a handstand is bound to fail due to a cramp in the agent's biceps. Does this deprive the agent of the specific ability to do a handstand here and now? Or does it just mask it? I take it that people will disagree about such questions.

For the possibilist, this should not come as a surprise. What it suggests is that there is actually no such thing as the correct understanding of specific abilities. The disagreements we have just considered can be solved only by resolving them. If we count only worlds as relevant in which absolutely everything, including the hyperacidity in the agent's muscles, is as in actuality, then it will be correct to say that the agent lacks the ability to do a handstand. If the hyperacidity is not among the facts we hold constant, we will correctly ascribe the ability to her; the hyperacidity will be a mere mask. Likewise in the first example. If the agent's fear is not among the relevant facts, we will ascribe the ability and count the fear as a mask. If it is, the fear deprives the agent of the ability.

Whether or not a specific ability is maskable will therefore depend on the comprehensiveness of the modal base. If absolutely every fact of the agent's situation is held fixed across modal space, then any impediment to the exercise of whatever act we are considering will itself have to be held fixed. Hence, the agent's ability to do a handstand is not masked by the agent's fear or a hyperacidity in her muscles, but annihilated. On this interpretation of a specific ability, then, specific abilities do not allow for masks. The modal base narrows down to exactly one world: the actual one.

If, in contrast, we are thinking of specific abilities in such a way that some facts of the agent's situation may still vary, then there is room for masks. For now we can abstract away from the fear and hyperacidity, say, and vary them across the possible worlds. And once we allow for that, the agent may very well come out as having the specific ability to do a handstand, despite her actual fear or her actual hyperacidity.

The picture this suggests is that abilities can be more or less specific, depending on just how comprehensive the set of facts is that determines the relevant worlds among which we then look out for a world in which the agent performs whatever act it is we are interested in. There are maximally specific abilities on one pole - for those abilities, we have to hold fixed absolutely every feature of the situation, be it ever so tiny. Along the scale, we can then align less specific abilities - abilities which allow for more and more variance of features of the agent's particular situation. The disagreement about the maskability of specific abilities can be resolved that way. Once we see that the two op- 
posing parties in the disagreement focus on varyingly extreme kinds of specific abilities, their disagreement vanishes into thin air.

This exposition has been quite simplistic, and much more would have to be said about a scalar understanding of general and specific abilities. Since I myself endorse a treatment of the distinction between general and specific abilities along very similar lines, we will have lots of time to turn to that task later on $(\rightarrow$ 4.5). For now, let's just note that the possibilist has a very interesting story to tell about the relation between general and specific abilities. In virtue of its appeal to varyingly comprehensive modal bases, it accommodates both and allows for a scalar picture of with maximally specific abilities on one pole, less than maximal, but yet specific abilities in the middle and general abilities towards the other end of the scale.

So much for the upside of possibilism with respect to the distinction between general and specific abilities and the understanding of masks. Now on to the downside. Although possibilism seems to capture one important aspect of the difference between general and specific abilities, we will now see that possibilism fails as an account of general abilities. More specifically, the problem is that the possibilist condition is not sufficient for the having of a general ability. It is much too easily met.

One way to approach the problem is to note that the modal base of ability statements is always realistic, according to the possibilist. Since what we hold fixed across modal space are always features of the actual world, the actual world is necessarily part of the modal base; it is always among the set of relevant worlds. To illustrate, suppose we are interested in an archer's ability to hit the bull's eye. If we are interested in her general ability, we hold certain features of the archer herself fixed: her brain structure, her vision, her muscular constitution maybe. What we do not hold fixed is her broken arm, say. If we are interested in the specific ability, we hold the broken arm fixed, too. Regardless of whether we are interested in the general or the specific ability, then, the relevant worlds will be those in which certain facts - states of affairs that obtain in the actual world - obtain. And of course, the actual world will be among those worlds. It will be a member of the set of worlds in which a certain subset of the actual facts obtain.

Obviously, this yields a problem. If the actual world is always among the relevant worlds, then any agent will automatically have the ability to do whatever she does in the actual world. To see that, just recall that to have an ability to $\phi$, according to the possibilist, is for there to be a relevant world in which the agent $\phi$ 's. And given that the actual world is a relevant world, this condition is obviously met if the agent $\phi$ 's in the actual world. 
The problem with this is that a singular successful performance of some act $\phi$, be it in the actual world or in any of the merely possible worlds, will rarely, if ever, suffice to show that an agent has a general ability to $\phi$. If I hit the bull's eye in the actual world, this does not show anything at all about my general ability to hit the bull's eye. This shows that I had the (maximally) specific ability to hit the bull's eye, certainly. But clearly, a singular success does not show that the agent has a general ability. This is a serious problem for the possibilist. The view apparently misses out on an important dimension here.

It is important to see that even though I have approached the problem from the assumption that the modal base always comprises the actual world, the problem itself has nothing to do with that particular feature of possibilism. Rather, it has to do with the existential quantification over the modal base quite generally. That is why I said in the beginning that the problem with possibilism is the modal force it assigns to ability statements. It is the single most essential feature of possibilism - the commitment to an analysis of abilities in terms of possibility - that causes the trouble.

To see that this is so, note that just as a singular successful performance of $\phi$ in the actual world suffices to make it true that the agent $\phi$ 's in a relevant world, a singular successful performance of $\phi$ in any other single one of the worlds that comprise the modal base is equally insufficient for an agent to have a general ability to $\phi$. Yet, possibilism predicts otherwise. Once there is a successful performance of $\phi$ to be found in the modal base worlds, the agent comes out as having the ability. Apparently, this is not a satisfactory account of general abilities.

The upshot of this section is mixed. On the one hand, possibilism clearly has a few merits over the simple conditional analysis in that it, first, offers an understanding of one important dimension on which general and specific abilities seem to differ and, secondly, yields an understanding of the workings of masks. On the other hand, possibilism does not really seem to capture what it is for an agent to have a general ability. The condition stated by the possibilist is not sufficient for an agent having a general ability.

\subsection{Upsides and downsides II - Impeded intentions, agentive vs. non-agentive abilities, and other possibilities}

In the last section, we saw that possibilism has one very important advantage over the simple conditional analysis. In this section, we will turn to a second feature that is both a merit and a problem for the view. Possibilism is very simple. It analyzes the ability to $\phi$ simply in terms of the possibility of $\phi$-ing and not, as the simple conditional analysis, in terms of a modal tie between the agent's in- 
tentions to $\phi$ and her performances of $\phi$-ing. Consequently, possibilism does not run into problems having to do with the postulation of such a tie. Specifically, it does not have the problems with impeded intentions or non-agentive abilities. This should not be overvalued, however. For as I will argue, possibilism may in fact be overly simplistic in that it fails to explain what it is that unifies abilities and sets them apart from mere physical possibilities, say.

That possibilism does not run into the problem of the impeded intention is easy to see. In cases of an impeded intention, recall, the agent is unable to $\phi$ in virtue of an impediment that prevents her from intending to $\phi$ in the first place. One of the examples I used to illustrate the problem was that of the coma patient (van Inwagen 1983: 119) who cannot raise her arm in virtue of being in a coma. The coma does not just prevent the agent from raising her arm, though, it also prevents her from intending to raise her arm to begin with. And this caused trouble for the simple conditional analysis.

Since the simple conditional analysis analyzes abilities in terms of a counterfactual tie between intentions and performances, to find out whether or not the agent has an ability, we had to move into the closest intention worlds and see whether or not the agent raises her arm there. But since the closest intention worlds are not, in fact, coma worlds, the counterfactual comes out true. If the agent intended to raise her arm, she would, because she would not be in a coma in that case. That is the wrong result.

The possibilist does not have the same problem. The possibilist restricts the possible worlds to the ones in which the relevant facts obtain. And among those, she checks for performance worlds. In virtue of this feature of the view, cases of impeded intentions are unproblematic. In the case of the coma patient, the coma will usually be among the relevant facts. Usually, we want to know what the coma patient can do in view of her coma. Is there an arm raising world among the so restricted worlds? Of course not. The modal base will neither contain arm raising worlds, nor will it contain any worlds in which the agent intends to raise her arm to begin with. The worlds the possibilist looks at all contain the very impediment that prevents both the intention to $\phi$ as well as $\phi$-ing. Thus, there will not be $\phi$-ing worlds among the relevant worlds. And hence, possibilism rightly yields that the coma patient and other agents whose intentions are impeded lack the ability to raise their arms, or whatever it is they cannot do in virtue of that impediment. Clearly, possibilism has an advantage over the simple conditional analysis here.

What about the good sense in which the coma patient can raise her arm, though? Recall that when we talked about impeded intentions in connection with the simple conditional analysis $(\rightarrow 2.3)$, we said that there is a good sense in which the coma patient retains quite a lot of her abilities - despite 
her coma. The ability to raise one's arm is not the best example to see that. But when you think of abilities that were acquired by a lot of training, like juggling, for instance, you will come to sense that there is a rather good sense in which one will want to say that the coma patient has retained that ability.

Possibilism has no trouble with any of this. In fact, it can account for both the sense in which one will want to say that the coma patient can, and the sense in which one will want to say that she cannot raise her arm, juggle, or whatever one is interested in. The key feature of possibilism here is the variance in the modal base. Sometimes, we are interested in what the agent can do in view of her coma. In that sense, she cannot do much. Sometimes, we are interested in what she can do in view of her amount of training in a certain discipline, or her muscular constitution, or a certain "programme in her brain", as Kratzer (1981: 54) has put it. In that sense, the coma patient has the ability to do quite a lot of things.

The possibilist can explain that smoothly. When we are interested in what the coma patient can do in view of her coma, the modal base will comprise only coma worlds. And among those, there is no arm raising world and no juggling world. But when we are interested in what the coma patient can do in view of her amount of training in something, the coma is varied across the possible worlds and the modal base will comprise all kinds of worlds in which the agent is not, in fact, in a coma. Among the so selected set of worlds, there will be quite a few in which the agent raises her arm and juggles. The possibilist is therefore apt to account for both senses of "having an ability" here - the sense in which the coma patient can, and the sense in which she cannot do certain things.

One last note on this, just to tie things back to the topics we have touched upon earlier. All this can also be put in terms of masks. Depending on how we select the modal base, the coma will either be a mask of the agent's ability to juggle, say, or it will deprive her of it. When the modal base comprises only coma worlds, the coma is an annihilator of the ability - the agent cannot juggle. When the coma is varied, the coma is a mask - the agent retains the ability to juggle, despite being in circumstances that conflict with the exercise of that ability. Things fall into place quite neatly here. Cases of impeded intentions are smoothly accommodated by possibilism.

Now on to non-agentive abilities. Here, too, possibilism looks more promising than the simple conditional analysis. Again, this has to do with the fact that intentions do not play a prominent role in the possibilist account. Non-agentive abilities, recall, include the ability to see, smell, or hear; to digest, dissociate, or produce saliva; to unintentionally read street signs or to understand something without listening. All of those things can, or can only, be exercised without a 
foregoing intention on the agent's part. This spelled trouble for the simple conditional analysis $(\rightarrow 2.6)$.

Possibilism does not have the same problem. According to the possibilist, an agent has an ability if and only if there is a relevant world in which the agent $\phi$ 's. This condition works equally well for non-agentive as for agentive abilities. An agent has the ability to bake a cake if and only if there is a relevant world in which she bakes a cake. An agent has the ability to produce saliva if and only if there is a relevant world in which she produces saliva. An agent has the ability to unintentionally read street signs if and only if there is a relevant world in which she reads street signs unintentionally. The fact that saliva is usually produced without a foregoing intention and street signs can only be read unintentionally, if no foregoing intention is formed, does not cause a problem at all for the possibilist. The modal base can comprise intention worlds and non-intention worlds alike. Intention worlds do not enjoy any special status.

So much, again, for some of the upside of possibilism. Let's now turn to a downside once more. The downside is that the simplicity of possibilism comes at a price. It is hard to see what unifies abilities and sets them apart from other restricted possibilities, such as mere physical possibility, say, on the possibilist framework. There is good reason to suspect that possibilism may in fact be a bit too simplistic; there seems to be an important dimension of what it is for an agent to have an ability that eludes the view.

Nomological possibility is possibility in view of the laws of nature, epistemic possibility is possibility in view of what is known, deontic possibility is possibility in view of what is allowed. Apart from those sharply contoured possibilities, there are other possibilities, of course. Possibility in view of certain facts of a situation, for instance. It may be possible in view of the security measures that a rollercoaster car derails, but not in view of the actual condition of the car and the rails. It may be possible in view of the closeness of a cliff that you fall off the mountain you are standing on, but not in view of your degree of carefulness.

If possibilists are right, then the possibility involved in the latter two possibility statements resembles that of ability ascriptions very closely. In both cases, we have existential quantification over a circumstantial modal base. Yet, "the car can derail" and "you can fall of the cliff" are not ability ascriptions, but rather ascriptions of what is often called a "physical possibility". A natural question to ask the possibilist is: what is the difference?

I don't think the possibilist has a fully convincing response to this question at hand. I guess what possibilists should reply is that the kind of possibility involved in ability ascriptions and statements like "the car can derail" and "you can fall off that cliff" is actually the same. That is the essence of their view about abilities, after all. They could then go on to try and characterize ability as- 
criptions in terms of other features of such ascriptions. One way one may try to do so is by characterizing ability ascriptions as statements that express restricted circumstantial possibility statements which relate agents to actions. "The car can derail" and "you can fall off that cliff" is not an ability ascription, according to this characterization, whereas "the president can start a war" is.

In view of the fact that there are such things as non-agentive abilities, however, it should be obvious that this characterization won't do. For possibilism to cover non-agentive abilities as well, the possibilist can hardly characterize ability statements by postulating that they relate agents to actions. Smelling, digesting, and reading street signs unintentionally are not (typically) actions. Yet, one can have the ability to do these things. Abilities will therefore have to be individuated differently, and it is hard to see how the possibilist is supposed to tackle this task with the tools available to her. Perhaps it can be done. But there is definitely an open question here.

\subsection{The problem with degrees and context sensitivity}

So far, we have noted both merits and problems of possibilism. In this and the next section, it will become obvious that the debits problems preponderate. In this section, we will see that possibilism fails to account for degrees and the corresponding kind of context sensitivity that attaches to ability statements. In the next section, we will see that the view is formally inadequate in that the most basic laws governing possibility fail to hold for ability statements.

Abilities, we said $(\rightarrow 1.3)$, come in degrees. Glenn Gould has the ability to play the piano to a higher degree than I do; my grandmother sews better, my cousin drives better, my father draws better than I do. One meteorologist may be better at predicting the weather than another. Two archers may be varyingly good at hitting the target.

Looking more closely at degrees of abilities, we saw that the degree of an agent's ability can be influenced on two dimensions: the degree of an ability depends, first, on the quality of the agent's performances and, secondly, on the range of circumstances across which the agent manages to deliver those performances. We called those two dimensions the dimension of achievement and the dimension of reliability respectively.

We also said that the gradable nature of abilities results in a specific kind of context sensitivity of ability ascriptions. That is because the degree of an agent's ability to $\phi$ required for the agent to count as having the ability to $\phi$ simpliciter varies across contexts. A darts player who misses $99 \%$ of the time, but manages to hit the bull's eye every $100^{\text {th }}$ time does not usually count as having the ability 
to hit it. There may be contexts in which we count her as having the ability, though. She has what it takes; it is within the range of what she can do. The context, we said, determines some threshold along the scale of degrees of abilities above which an agent counts as having the ability simpliciter. Once we have degrees, then, we also have the mechanism for the corresponding context sensitivity.

The problem with possibilism is that it fails to account for degrees and, consequently, for the corresponding kind of context sensitivity of ability ascriptions. As in the case of the simple conditional analysis, the problematic cases are not so much the ones in which achievement is the dimension which matters, even though they, too, require a supplementation of the possibilist framework as it stands. To see that, reconsider the driving abilities of my partner and his friend. Both of them can drive a car, but while my partner is a very good driver, his friend drives poorly. At first sight, this is a problem for the possibilist. The possibilist holds that an agent has an ability to $\phi$ if and only if there is a relevant world in which the agent $\phi$ 's. And obviously, this is true for my partner and his friend alike. His friend can drive, after all. It is thus possible, given her amount of training and so forth, that she drives. The problem is that she is not very good at it. So, her driving performances will be of rather low quality. As it stands, possibilism does not have the means to account for this kind of difference between two agents.

Of course, the possibilist framework can easily be supplemented by an account of degrees that track variances on the level of achievement. The possibilist can avail herself of a strategy analogous to the one I suggested in connection with the simple conditional analysis $(\rightarrow 2.5)$. She can attach values to the quality of an agent's performance and postulate that the degree of an agent's ability is higher the higher that value is. Were achievement the only dimension that counted, an account of degrees of abilities would be quite easily available for the possibilist.

But achievement is not the only dimension that counts. To come up with a comprehensive account of degrees of abilities, variances on the dimension of reliability will have to be accounted for as well. This is where possibilism exhibits a deep structural shortcoming. Reconsider Ted and Fred, our two meteorologists. Fred has developed an algorithm, by means of which he can predict $99 \%$ of all weather constellations correctly. Ted has developed a faulty algorithm - it goes wrong for $45 \%$ of all weather constellations. Clearly, Fred is the better meteorologist. Possibilism, however, has no means to distinguish between the degrees of Ted's and Fred's weather prediction abilities. For both, there will be relevant worlds in which they predict the weather correctly. According to the possibilist, the two are on a par. 
The problem with possibilism is that the existential quantifier does not seem to be particularly well-suited to account for degrees. Once there is one performance world among the relevant worlds, the possibilist condition is satisfied. There does not seem to be any room for the kind of scale that would have to be established in order to account for differences on the dimension of reliability.

But wait a minute! Did we not talk about degrees of possibility earlier on? And wasn't there a tool that Kratzer employed in her semantics to account for such degrees? - The tool that comes to mind here, of course, is the ordering source I mentioned when I first introduced the standard semantics of "can" statements $(\rightarrow 3.1)$. And indeed, it seems to be just the right means for the purpose at hand. It is designed to account for degrees of possibility. Why not use it in order to account for degrees of ability, too?

Unfortunately, the ordering source does not do the job. The ordering source, recall, ranks the possible worlds according to some ideal. One very common ordering source is a stereotypical one. In the stereotypical ideal, everything is normal and the possible worlds are then ranked in accordance to their similarity to that ideal - the more normal a world is, the closer it comes to the stereotypical ordering source. We saw that a stereotypical ordering source is what is at work when it comes to the detective's judgement that there is a better possibility of Kim being the murderer than of Jim being the murderer. Each of them can be the murderer, but the closest worlds in which Kim is the murderer are much closer to the ideal of normality than the closest worlds in which Jim is the murderer.

So far, so good. The crucial question is: can the same kind of strategy be employed for degrees of ability? Is there an ordering source, such that the closest worlds in which Fred predicts the weather correctly are always closer to the ideal than the closest worlds in which Ted predicts the weather correctly? At first, it may seem plausible that the stereotypical ordering source is already the right one for the purpose at hand. For are not worlds in which Fred predicts the weather correctly more normal than worlds in which Ted predicts the weather correctly? After all, Fred is far more reliable in his weather predictions than Ted.

But we need to be careful. What we need to ask is: what would be the case in the most normal world imaginable? Then we have to find out how close to that world the closest world is in which Fred predicts the weather as opposed to the closest world in which Ted predicts the weather. The answer suggests that the stereotypical ordering source will not do the job of telling Fred's and Ted's respective degrees of the ability to predict the weather apart. For it seems that in the most normal world imaginable, both Fred and Ted will predict the weather correctly. Both have a quota of correct predictions that exceeds 50\%, after all. Thus, it would seem that a world in which both agents predict the weather cor- 
rectly is, ceteris paribus, more normal than any world in which one of them makes a wrong prediction. ${ }^{4}$ Hence, the closest worlds in which Fred makes a right prediction is just as close to the ideal as the closest world in which Ted makes a right prediction.

From here, we can move in two directions. We can either try and come up with all kinds of ordering sources and see whether one of them is suited to account for degrees of agents' abilities. Or we can think about the prospects of solving the problem of degrees by the use of ordering sources quite generally. I suggest we do the latter. The ordering source as such fails to provide a proper means to account for degrees of ability.

To see that, note that reliability is a matter of performances across various situations - it is a matter of a quota. If I am more reliable than you are in hitting a target, then this has to do with my hitting the target across a wider range of circumstances. The possibilist, however, can only ever take one world into account. Even if we rank the worlds in accordance to some ideal, then, - stereotypical or of any other kind - we will still have to judge the agent's degree of ability on the basis of her performance of just one world, namely that which comes closest to that ideal. Given the nature of reliability - its tight connection to the idea of a quota - this strategy seems faulty in a very substantial way. No matter what kind of ranking we impose on the possible worlds, the fact that there is a difference between two agents in the worlds closest to the ideal will never ensure that this difference mirrors the agents' differences in terms of some relevant quota across worlds. ${ }^{5}$

\subsection{A formal problem}

Up to this point, several doubts concerning the viability of a possibilist account of abilities have accumulated. Possibilism is unable to set abilities apart from mere physical possibilities $(\rightarrow 3.4)$, it fails to account for general abilities $(\rightarrow$ 3.3), and it sits more than awkwardly with the idea of degrees of ability ( $\rightarrow$ 3.5). In this section, we will confirm the impression that there is indeed something wrong with the view. As I will lay out in what follows, possibilism is actually formally inadequate. There are formal considerations which show rather clearly that the "can" of ability is not the "can" of possibility.

4 We can stipulate that the predictions of both agents are better the more normal the weather conditions are.

5 Vetter (2015) argues against a possibilist understanding of degrees of dispositions along the same lines. 
According to the possibilist, the "can" of ability functions as a restricted possibility operator. But as Kenny (1976) has convincingly shown, the "can" of ability cannot be accounted for in terms of a possibility operator, because the "can" of ability does not obey the most fundamental laws of the logic of possibility. Since the possibility operator is defined by the formal rules that govern it, this is a knock-down argument. It just does not make any sense to claim that the "can" of ability is a restricted possibility operator, if none of the most basic principles of the possibility operator apply. Let us go through Kenny's argument to see how it works.

Suppose the possibilist is right in her verdict that ability statements, such as "S can $\phi$ ", express something along the lines of "It is possible (in some properly restricted sense) for S to $\phi$ ”. Then the logical form of ascriptions of ability ascriptions would be CAN(S ф's), where CAN is a properly restricted possibility operator. If CAN is a possibility operator, then it will have to be construed as a sentence operator. And the sentence it ranges over will express a proposition according to which some subject engages in some doing, paradigmatically an action. All of that should be familiar from the section on the basic Kratzer semantics $(\rightarrow 3.1)$. On this construal, ability ascriptions would be akin to other forms of restricted possibility statements, such as deontic, nomological, or epistemic possibility claims.

The problem Kenny notes is that ability statements do not exhibit the right kind of logic to pass for possibility statements; they fail to obey the most basic laws of possibility. To see that, note that the weakest system of modal logic suitable for alethic interpretations of the operators is the system KT. One axiomatization of KT contains (among other principles) the axiom schemata

$$
\begin{aligned}
& \text { T. } p \rightarrow \diamond(p) \\
& \text { K. } \diamond(p) \vee \diamond(q) \leftrightarrow \diamond(p \vee q)
\end{aligned}
$$

The validity of these two principles can be derived directly from the possible worlds semantics of the possibility operator. In possible worlds terminology, the T-axiom reads: if $\mathrm{p}$ is the case in the actual world, then there is some world in which $\mathrm{p}$ is the case. That is clearly valid, because the actual world, of course, is some world.

The K-axiom can be split up into two conditional claims. From left to right it states that

K1. $\diamond(p) \vee \diamond(q) \rightarrow \diamond(p \vee q)$

From right to left it states that 
K2. $\diamond(p \vee q) \rightarrow \diamond(p) \vee \diamond(q)$

In possible worlds terminology, K1 reads: if there is some world in which $\mathrm{p}$ is the case or there is some world in which $q$ is the case, then there is some world in which p or q is the case. Apparently, this is a logically valid principle, for "p or q" will hold both in any p-world and in any q-world. Hence, if there is some p-world or some q-world, eo ipso, there will be some p-or-q-world.

Likewise for $\mathrm{K} 2$. In possible worlds terminology, the principle reads: if there is some world in which p or $q$ is the case, then there is a world in which $p$ is the case or there is a world in which $q$ is the case. Again, this principle is clearly valid. Since any p-or-q world is either a p-world or a q-world, there being a $\mathrm{p}$ or-q-world implies that there is a p-world or a q-world.

As Kenny points out, neither of these schemata is correct, if the possibility operator (the diamond) is interpreted as the "can" of ability. His arguments proceed by counterexample. Let's start with the T-axiom $\mathrm{p} \rightarrow \diamond \mathrm{p}$. Interpreting the possibility operator in terms of the "can" of ability, the theorem states that

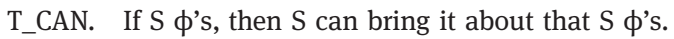

Note that I am using the formulation "S can bring it about that S ф's". This locution has the purpose of reflecting, in ordinary language, that the "can" of ability functions as a sentence operator on the possibilist's framework. Nothing substantial hinges on this. It just helps to integrate natural language ability talk into the schemes of the modal laws governing possibility.

On the face of it, T_CAN looks like a valid principle. As Geach points out, it seems to be a truism that "what a man does he can do; that is clear if anything in modal logic is” (1957: 15). And according to Mele,

there is an ordinary sense of 'able' according to which agents are able to do whatever they do. In this sense of 'able', an agent's having A -ed at a time is conceptually sufficient for her having been able to A. (Mele 2002: $447 \mathrm{f}$.)

Suppose Geach and Mele are right. Suppose, that is, that there is, in fact, an ordinary sense of the "can" of ability which is in line with axiom T. ${ }^{6}$ The problem is just that there are other senses of the "can" of ability which are clearly not. For

6 You may struggle with the idea that there is such a sense. I do not want to commit on this issue. As it will turn out, my own view has the resources to account for a sense of "specific ability", according to which a subject has the specific ability to do whatever she did. But my view does not commit me to endorsing this sense ( $\rightarrow 4.5$, b \& d). 
general abilities, for instance, the principle clearly fails. As Kenny points out, for instance,

"[a] hopeless darts player may, once in a lifetime, hit the bull, but be unable to repeat the performance because he does not have the [general] ability to hit the bull. (...) Counterexamples similar to [this] will always be imaginable whenever it is possible to do something by luck rather than by skill. But the distinction between luck and skill is not a marginal matter in this context: it is precisely what we are interested in when our concern is [general] ability, as opposed to logical possibility or opportunity" (Kenny 1976: 214).

What Kenny points out here is that the having of a general ability requires more than just an individual success. This insight is not new; I emphasized the same fact in the section about general and specific abilities ( $\rightarrow 3.3$ ), when I argued that possibilism fails to account for general abilities. The new twist to this insight is that possibilism is therefore at odds with the logic of possibility. The T-theorem, while valid for all established alethic interpretations of the possibility operator, is clearly invalid for the "can" of general ability. This fact casts additional doubt upon any view according to which the "can" of general ability is understood in terms of the possibility operator.

Let's now turn to the K-axiom, which seems likewise problematic, if the diamond is interpreted as the "can" of ability. As Kenny points out, it fails in both directions, but in what follows, I will focus on K2. ${ }^{7}$ Inserting the "can" of ability for the diamond, $\mathrm{K} 2$ reads

K2_CAN. If S can bring it about that S ф's or $\psi$ 's, then S can bring it about that S $\phi$ 's or S can bring it about that $S$ 's.

On the face of it, this, too, looks like a valid principle, and indeed, it has many true instances. If I can bring it about that I ride my bike or drive my car, then I can bring it about that I ride my bike or I can bring it about that I drive my car. If I can bring it about that I eat an apple or a banana, then I can bring it about that I eat an apple or I can bring it about that I eat a banana.

7 Interpreting the diamond as the "can" of ability, K1 states that if S can bring it about that S $\varphi$ 's and $S$ can bring it about that $S \psi$ 's then $S$ can bring it about that $S$ either $\varphi$ 's or $\psi$ 's. This is false, as can be seen when $\psi$ is non- $\varphi$. The president has the ability to bring it about that the president destroys Moscow and he has the ability to bring it about that the president does not destroy Moscow. Yet, he doesn't have the ability to bring it about that the president destroys Moscow or the president does not destroy Moscow. Why does he lack that ability? Because (The president destroys Moscow or the president does not destroy Moscow) is a necessity, and according to Kenny no one has the ability to bring about a necessity. 
But there are problem cases. Suppose I am faced with a stack of cards that is turned face-down (Kenny 1976: 215). In that case, I have the general ability to pick red or black. Yet, it seems, I neither have the general ability to pick red nor do I have the general ability to pick black. It is therefore false that

\#. If S can bring it about that S picks black or red, then S can bring it about that S picks black or S can bring it about that $\mathrm{S}$ picks red.

For an even better example, suppose I have the general ability to hit the dart board (ibid: 215f.). This implies that I have the general ability to hit the upper or the lower half of the dart board. ${ }^{8}$ Yet, I may well lack the general ability to hit the upper half of the bull and also lack the general ability to hit the lower half. It is therefore false that

\#\#. If $\mathrm{S}$ can bring it about that $\mathrm{S}$ hits the upper half or $\mathrm{S}$ hits the lower half, then $\mathrm{S}$ can bring it about that $\mathrm{S}$ hits the upper half or $\mathrm{S}$ can bring it about that $\mathrm{S}$ hits the lower half.

Why is this an even better counterexample to K2_CAN? Because it can be strengthened. The example works better the more discriminatory we get. That I can hit the dartboard implies that I can hit one square-millimeter on the board, or another, or another, and so on. But it obviously does not imply that I can hit any particular square-millimeter on the board.

The important point is that in both examples, the left-hand side of the conditional is satisfied while the right-hand side is not. And, as Kenny points out, "[s]imilar counterexamples can be constructed in connection with any other discriminatory skill" (ibid. 215). Kenny has a very strong point here. The "can" of ability disobeys the rules of the possibility operator. This makes it almost impossible to cling to the view that the "can" of ability is a "can" of possibility.

\subsection{Upshot}

Let's go through the main line of thought of this chapter once more. In this chapter, we looked at possibilism - the view that an agent has an ability to $\phi$ if and only if it is restrictedly possible for the agent to $\phi$; or, to put it in the possible

8 If you don't like the inference from "having the ability to hit the board" to "having the ability to hit the upper half or having the ability to hit the lower half", never mind. We can run the argument by assuming right away that the agent has the latter ability. 
worlds terminology we used throughout the chapter, if and only if there is a relevant world in which the agent $\phi$ 's. We noted quite a few merits about possibilism, but also a number of problems, which together showed very clearly that abilities are not, in fact, restricted possibilities.

Let's start with the merits. First of all, possibilism seemed much better suited than the simple conditional analysis in making sense of the distinction between general and specific abilities $(\rightarrow 3.3$ ). The crucial tool here was the variance in the set of worlds that go into the modal base: having a general ability, on the possibilist framework, is for there to be a $\phi$-ing-world among one set of worlds, whereas having a specific ability is for there to be a $\phi$-ing-world among a different set of worlds. When I have a broken leg, say, I still have the ability to dance in view of the "dancing program" in my brain, but not in view of my broken leg. And depending on whether or not we hold the broken leg or merely the dancing program fixed, there either will or won't be a world among the thus-restricted set of worlds in which I dance. This looks very promising at the outset and seems to capture a very important dimension on which general and specific abilities differ.

By the same token, possibilism allows for an understanding of the workings of masks ( $\rightarrow$ 3.3). The broken leg is a mask for the general ability to dance. It is a fact in the world that would interfere with the exercise of the ability. Yet, it does not deprive the agent of the ability, because it is not among the set of facts that are held fixed across the possible worlds. When we are interested in the general ability to dance, we are not interested in the ability to dance in view of one's currently broken leg, but rather in the ability to dance in view of the dancing program in one's brain or one's overall bodily constitution, or what have you. Yet, the broken leg clearly deprives us of the more specific ability to dance in view of one's broken leg. Here, the broken leg is held fixed, and hence there is no dancing world among the modal base worlds. Possibilism explains all this very smoothly.

The third merit we noted was that possibilism does not run into the problem of the impeded intention $(\rightarrow 3.4)$. The coma patient cannot raise her arm, on the possibilist framework, because the coma will have to be held fixed across the possible worlds and hence there will not be any arm raising worlds among the modal base. Since the possibilist does not put emphasis on the link between intentions and performances, there is no need to move beyond the coma worlds in order to reach the closest intention worlds. Instead, the possibilist checks for performance worlds among the coma worlds. And voilà: there are none. This is the right result. Again, that is not to say that there is no sense in which the coma patient retains certain abilities. The possibilist can account for both senses, depending on whether or not the coma is held fixed. 
Fourth, and relatedly, possibilism does not run into problems with nonagentive abilities ( $\rightarrow$ 3.4). Since abilities, quite generally, are understood in terms of the possibility of performance, non-agentive abilities do not pose any particular problem. Having the ability to dance is for it to be possible for the agent to dance. Having the ability to produce saliva is for it to be possible for the agent to produce saliva. Since no emphasis is put on the tie between intentions and performances, no problem emerges from abilities that can or can only be exercised without a foregoing intention on the agent's part.

But there were also some severe problems with the view.

First, there was a problem with general abilities $(\rightarrow$ 3.3). Possibilism may shed light on one dimension on which general and specific abilities differ, but it certainly fails as an account of general abilities. Having a general ability to $\phi$ seems to require a certain quota of success in $\phi$-ing. There being just one world (actual or possible) in which the agent $\phi$-s certainly isn't enough. The last word on general and specific abilities obviously has not been spoken by the possibilist.

Secondly, the possibilist fails to properly delineate abilities from other possibilities, like physical possibilities $(\rightarrow 3.4)$. What is the difference between a statement of the form "I can dance" or "I can read street signs unintentionally" and a statement of the form "You can fall off the cliff"? All three express restricted circumstantial possibility. You and I are both agents. And reading street signs unintentionally is no more an action than falling off a cliff is. This indicates that possibilism is actually a bit too simplistic. There is something about abilities that sets abilities apart from physical possibilities. And whatever that feature is, it does not seem to be properly captured by the possibilist.

Thirdly, there was the problem with degrees and the corresponding kind of context sensitivity that attaches to ability statements $(\rightarrow 3.5)$. The problem with possibilism is that the existential quantifier does not seem to be particularly well-suited to account for degrees. Once there is one performance world among the relevant worlds, the possibilist condition is satisfied. There does not seem to be any room for the kind of scale that would have to be established in order to account for differences of degrees that trace back to differences on the dimension of reliability - i.e. the range of circumstances in which the agent manages to deliver a performance. The ordering source does not provide help here. Degrees of abilities fail to be accounted for by the possibilist. As a consequence, the context sensitivity of ability statements that results from the gradable nature of abilities remains mysterious as well.

Fourthly, there was the formal problem with the possibilist's core claim: ability statements do not express restricted possibility statements, because ability statements do not obey the most fundamental logical laws governing possibility 
statements. Since the possibility operator is defined by the laws that govern it, this is a decisive problem. Possibilism is deeply flawed. 